\title{
SURFING WAVES GENERATED BY A HULL
}

\author{
M. A. de Schipper ${ }^{1}$, S. de Vries $^{1}$, M. Henriquez ${ }^{1}$, A.J.H.M. Reniers ${ }^{1,3}$ \\ H.J. de Koning Gans ${ }^{2}$, R.J. Labeur ${ }^{1}$ and M.J.F. Stive ${ }^{1}$
}

\begin{abstract}
In this paper a new wave pool concept is presented using ship hulls as wave generators To evaluate the potential of such a concept a towing tank experiment is conducted to investigate ship wave generation in a confined space. In the towing tank experimen various hull sizes and velocities are examined to maximize the generated wave height The laboratory data shows that when blocking of the cross section of the pool by the hul is large, the large return flow significantly influences the wave height. This is in contrast to the open water case where the return flow is much smaller and therefore is not incorporated in wave height predictions. Based on the results of the physical model tests an optimum hull size and velocity are derived to maximize the potential use for surfing and a preliminary design of the wave pool is proposed.
\end{abstract}

\section{INTRODUCTION}

Surfing as a sport is growing. Locations with good surfing conditions are limited and therefore crowded. The growing interest for the sport has led to the development and construction of several artificial surf pools worldwide.

Traditionally these surf pools are of rectangular shape and use wave maker caissons along an outer edge to generate waves. Water inside the caisson is pumped above the still water level, which when released, forms a wave. The generated wave propagates over a curved bottom topography and breaks in a peeling manner, providing a moving breakpoint to surf along.

This study presents a new concept for wave generation as envisioned by G. Webber. Waves are generated by towing ship-like hulls along the side wall of a pool. The waves generated by the hull, called ship waves from hereon, propagate from the hull and break on a sloping bottom at the opposite side of the pool. If this wave generation concept is applied to a circular pool with a large diameter $(\sim 200 \mathrm{~m})$, it would theoretically be possible to surf continuously (Fig. 1).

The ultimate goal of a wave pool is to mimic good surf conditions as found along the coast. The basic characteristics of good surfing waves have been

\footnotetext{
${ }^{1}$ Faculty of Civil Engineering and Geosciences, Section of Hydraulic Engineering, Delft University of Technology, Stevinweg 1, Delft, 2628 CN, The Netherlands.

${ }^{2}$ Faculty of Mechanical, Maritime and Materials Engineering, Section of Ship Hydromechanics and Structures, Delft University of Technology, Mekelweg 2, Delft, 2628 CD, The Netherlands.

${ }^{3}$ Rosenstiel School of Marine and Atmospheric Science (RSMAS), University of Miami, 4600 Rickenbacker Causeway, FL 33149-1098, Miami, USA
} 
described by Hutt et al. (2001) and Walker (1974) and these provide the requirements for the current project.

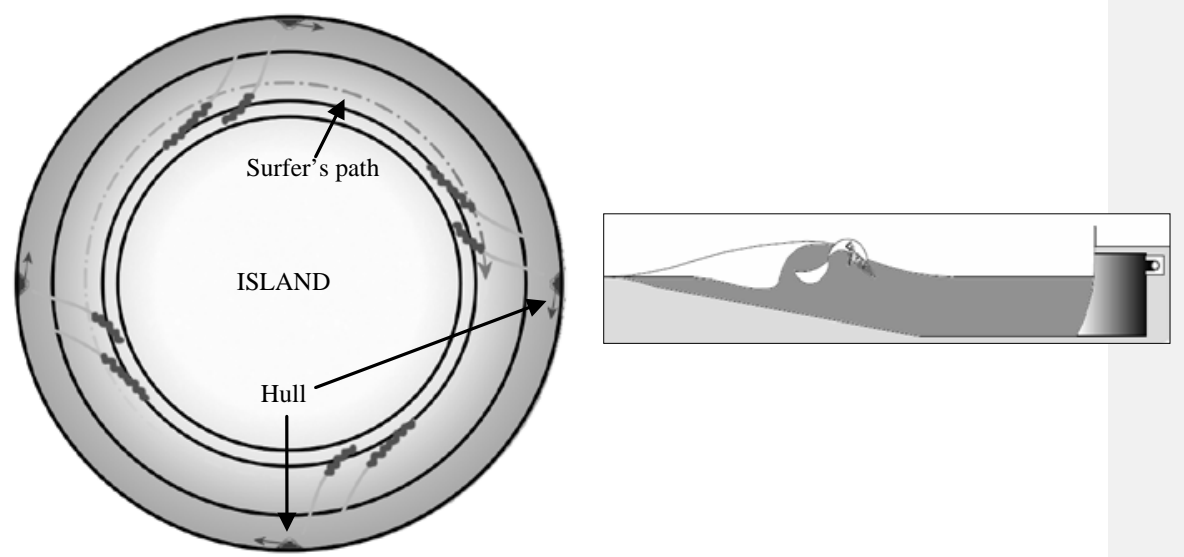

Figure 1. Top view and cross section of wave pool concept.

The quality of the wave, and therefore the skill required to surf it is expressed in three parameters; the wave height, peel angle and breaker shape. To accommodate intermediate to expert surfers, a plunging wave height of $2 \mathrm{~m}$ combined with peel angles between $40^{\circ}$ and $60^{\circ}$ are required.

This paper focuses on wave generation by blunt hulls in a confined space with the intention to maximize the generated wave height. First the physical background will be given to clarify the forthcoming results of a towing tank experiment. Next, current state-of-the-art numerical models will be evaluated using the towing tank data and the paper will conclude by a preliminary design of the wave pool

\section{PHYSICAL BACKGROUND}

The desired wave height of $2 \mathrm{~m}$ implies that a large amount of energy has to be transferred by the hull into a relatively small water mass. The water motion around a hull in an enclosed environment like a small canal can be subdivided into a primary and secondary wave pattern. In the following section both wave types will be elaborated for the wave pool case. To investigate the wave generation the circular basin is initially simplified to a straight canal.

\section{Primary wave}

The primary wave of a hull in a canal concerns a water level depression $\boldsymbol{z}_{\boldsymbol{a}}$ and the corresponding return current $U_{r}$ alongside the hull. Consider a hull moving through a rectangular canal, as depicted in Fig. 2. 
The velocity of the hull is then commonly expressed in a non dimensional form as the Froude depth number $F_{r, d}=\frac{V_{h}}{\sqrt{g h}}$, where $V_{h}$ is the hull velocity with respect to the bank, $h$ is the water depth and $g$ is the acceleration of gravity.
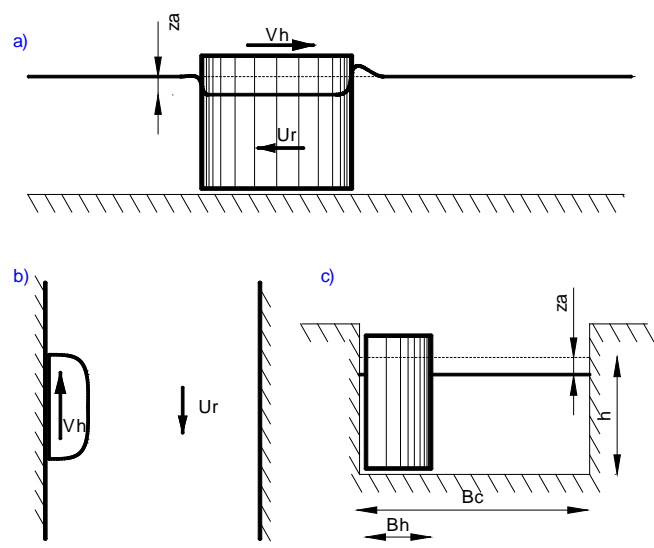

Figure 2. Schematic overview of primary wave pattern; a) side view of the hull, b) top view and c) cross section of the canal.

However since return current $U_{r}$ is present alongside the hull, the velocity of the hull with respect to the water flowing alongside is $V_{h}+U_{r}$. Or, in non dimensional form as a Froude number relative to the return flow $F_{r, d}^{*}=\frac{V_{h}+U_{r}}{\sqrt{g h}}$.

The magnitude of $z_{a}$ and $U_{r}$ can be estimated using mass and momentum conservation. Taking the reference frame on the moving hull leads to the following set of equations (assuming incompressibility of water, and no energy losses due to friction or turbulence).

$A_{c} V_{h}=\left(A_{c}-A_{s}-Z_{a}\left(B_{c}-B_{s}\right)\right)\left(V_{s}+U_{r}\right)$

$\frac{1}{2} \rho V_{h}^{2}+\rho g h=\frac{1}{2} \rho \alpha_{u}\left(V_{h}+U_{r}\right)^{2}+\rho g h\left(h-z_{a}\right)$

Where $A_{c}$ and $A_{s}$ are the cross sectional areas of the canal and the ship, $B_{c}$ is the canal width, $B_{h}$ is the width (beam) of the hull, $\rho$ is the volumetric density of the water and $\alpha_{u}$ is the non-dimensional coefficient accounting for the non 
uniform velocity distribution in the canal cross section alongside the hull $\alpha_{u}=1.4-0.4 F_{r, d}$ (Jansen and Schijf 1953).

One single equation can be obtained by combining Eq. 1 and Eq. 2, as shown by Lap (1954):

$$
\frac{V_{h}}{\sqrt{g h}}=\frac{\left(V_{h}+U_{r}\right)}{\sqrt{g h}}\left\{1-\frac{A_{s}}{A_{c}}-\frac{\alpha_{u} \beta}{2}\left(\left(\frac{\left(V_{h}+U_{r}\right)}{\sqrt{g h}}\right)^{2}-\left(\frac{V_{h}}{\sqrt{g h}}\right)^{2}\right)\right\}
$$

Or in non dimensional form:

$$
F_{r, d}=F_{r, d}^{*}\left\{1-K-\frac{\alpha_{u} \beta}{2}\left(F_{r, d}^{*}{ }^{2}-F_{r, d}{ }^{2}\right)\right\}
$$

Where $K$ the blockage factor given by $\frac{A_{s}}{A_{c}}$ and $\beta$ is the relative width of the free surface $\left(\frac{B_{c}-B_{h}}{B_{c}}\right)$. A self propelled ship will sail at velocities much smaller than $F_{r, d}=1$ and the return flow can be calculated using Eq. 4. For hull velocities leading to $F_{r, d}=1$ it is impossible to comply to both Eq. 1 and Eq. 2. At this point that the flow beside the hull becomes critical $\left(F_{r, d}^{*}=1\right)$. Within this so called trans-critical range the cross-section alongside the hull is too small for the discharge calculated with Eq. 1, given a certain energy head. Flow velocities alongside the hull become critical and the surplus of discharge is piled up before the hull. A positive translation wave precedes the hull and a smaller negative wave of translation follows the hull as a consequence (Fig. 3). In the so-called subcritical range, where ships sail in general, no translation wave is present (Fig. 2a).

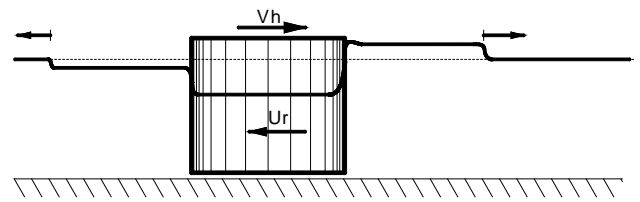

Figure 3. Schematic overview of side view of the hull in trans-critical conditions

The onset of the trans-critical range is a function of the blockage $K$ and can be calculated using Eq. 4. In case of a large hull in a narrow channel, blockage is high and the trans-critical domain starts at relatively small velocities. On the other hand a vessel sailing in open water has no lateral boundaries and hence virtually no trans-critical range. 


\section{Secondary wave}

The pressure gradients in the flow close to the hull act as a point disturbance. Circular wave patterns radiate out from such a disturbance point, similar to a stone thrown into a pond. These circular wave patterns are continuously generated while the hull propagates along its sailing line and superposition of these circular patterns gives a V-shaped interference pattern, as shown in Fig. 4.

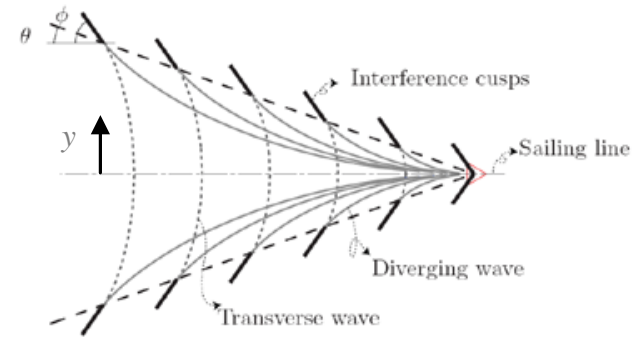

Figure 4. Theoretical secondary wave pattern in deep water

The V-pattern consists of interfering diverging and transverse waves. The resulting interference cusps (called featherlet waves) are located on this interference line. In deep water (roughly when $F_{r, d} \leq 0.6$ ) the wave angles $\theta$ and $\varphi$ are independent of hull shape and speed and measure $19^{\circ}$ and $55^{\circ}$ with the sailing line respectively. Wave lengths and periods can be calculated from linear wave theory (Stoker 1957).

In shallow water, restrictions in wave energy propagation speed causes the angles $\theta$ and $\varphi$ to increase up to $90^{\circ}$ at $F_{r, d}=1$ and a shock wave is formed (De Schipper 2007).

The height of the interference cusps has been the subject of various research projects. None of these however resulted in a generic theoretical relation for hull shape and velocity versus the wave height. Verheij and Bogaerts (1986) give a review of the outcomes and present several equations and tuning coefficients based on experimental data. Generally speaking the observed wave height at location $y$ from the ship is a function of the ship's geometry, water depth $h$, velocity $V_{h}$. Cusp heights for deep water ship waves $\left(\theta=19^{\circ}\right)$ are given by,

$\frac{H}{h}=\alpha_{1}\left(\frac{y}{h}\right)^{-1 / 3}\left(F_{r, d}\right)^{\alpha_{2}}$, 
where $y$ is the distance to the bow perpendicular to the sailing line ( $y>$ $0), \alpha_{1}$ is the non dimensional hull shape coefficient and $\alpha_{2}$ is the exponent giving the relation between non dimensional waveheight and velocity.

Verheij and Bogaerts (1986) show that for blunt shapes $\alpha_{1}=1.0$ and $\alpha_{2}=4.0$ give the best agreement with laboratory data. The equation does not hold for large Froude depth numbers since in this case wave angles are beyond $19^{\circ}$ and the wave height in the region $F_{r, d}$ larger than 0.8 is overestimated.

The waves used for surfing in the wave pool concept are the secondary waves. They are more suited for surfing since these free surface waves can travel further from the object and approach the shore at an angle. The oblique incidence of waves is one of the key aspects of surfable waves. The primary wave on the other hand causes large return flow velocities and has to be minimized. Furthermore the trans-critical regime has to be avoided. The translation wave in front of the hull elongates until it starts to break. Since the wave breaking introduces a large amount turbulence, the wave field behind the hull will deteriorate in the presence of a translation wave.

\section{PHYSICAL MODEL STUDY}

A towing tank experiment was conducted to determine the influence of the water level depression and return flow (the primary wave) on the generation of the secondary wave field as well as to investigate under which conditions the largest secondary waves are generated.

Within an $80 \mathrm{~m}$ towing tank, the influence of different hull widths, drafts and velocities was examined. An array of 4 resistance type wave gauges and one electromagnetic current meter were installed to record the return current velocity and the surface elevation and its distribution. Wave gauge signals consist of the primary wave system and the secondary wave system imposed on each other. These two 'intrinsic modes' were separated using an approach based on Empirical Mode Decomposition (EMD) (Huang et al. 1996) and analysed separately. The decomposed primary and secondary wave signals are depicted in Fig. 5.
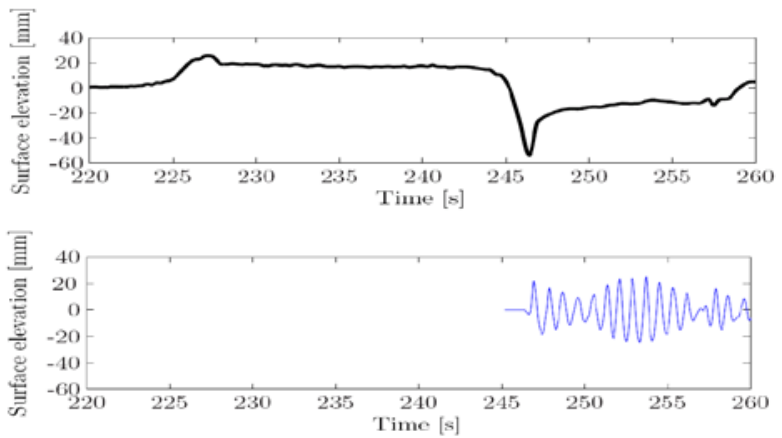
Figure 5. Wave gauge signal after empirical mode decomposition. Hull passage is around $\mathrm{t}=\mathbf{2 4 6} \mathrm{s}$. Primary wave field including the translation waves (top) and secondary wave field (bottom). Note that the secondary wave period decreases soon after hull passage.

Results

Over 80 different towing tank runs were examined and primary and secondary wave fields were compared to the previously cited theoretical values. Visual observations show that the secondary wave field is to a large extent affected by the primary wave field. In the subcritical regime the flow velocities alongside the hull are relatively small and concentrated close to the hull. The secondary wave field can develop and forms the predicted V-shaped pattern. In the trans-critical regime the flow is strongly contracted and the strong divergence of the flow after the hull passage generates an (undulating) bore. In this highly non-uniform and turbulent flow field the secondary wave field cannot develop and is difficult to observe.

The presence of a translation wave marks this trans-critical domain. The laboratory data was examined to asses whether the onset of the trans-critical regime can be predicted using Eq.4. The fastest sub-critical run (without translation wave) and the slowest trans-critical run (with translation wave) are selected for each hull shape and the onset of the trans-critical regime is assumed to be the average of fastest subcritical and slowest trans-critical velocity. These are compared in Fig. 6. Although Eq. 4 is based on a highly schematized setup (no friction or turbulence losses), it forms a good fit with the laboratory data.

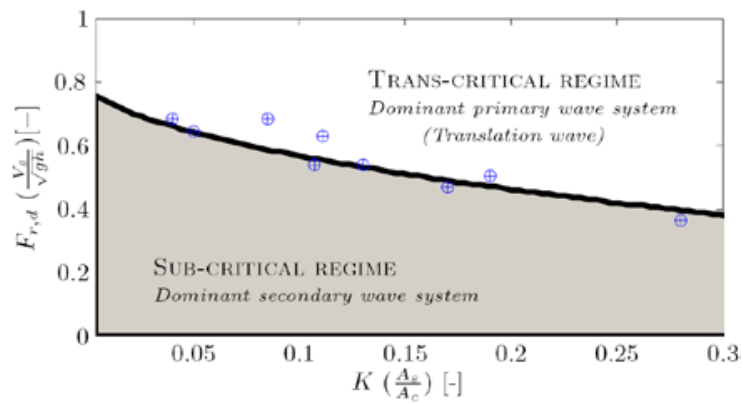

Figure 6. The onset of the trans-critical regime as found during the experimental study. Symbol $\oplus$ represents the towing tank data, thick line obtained using Eq. 4. Grey area is the sub critical domain.

The measured secondary wave field is highly influenced by the return current velocity. If the wake is stationary to the hull, wave angles and wave periods are by definition larger in regions with large return currents. Soon after the hull passage, the return current is large. The increase of the wave period due to this return current soon after the hull passage can be seen in Fig. 5 . 
Detailed examination of the wave periods using wavelet analysis shows that the wave periods correspond well to linear theory, provided that the hull velocity is taken relative to the surrounding water, including the return current.

Wave heights are slightly underestimated by Eq. 5, but a reasonable correlation is obtained $\left(R^{2}=0.6\right)$. Since the water level depression and return velocity have proven to influence the secondary wave field considerably it is proposed to adjust Eq. 5 to incorporate the local flow regime:

$\frac{H}{h-z_{a}}=\alpha_{1}\left(\frac{y}{h}\right)^{-1 / 3}\left(F_{r, d}^{*}\right)^{\alpha_{2}}$,

where the LHS represents the dimensionless wave height and $F_{r, d}^{*}$ is the Froude number in the wave generation area in the vicinity of the hull. Wave decay term $(y / h)^{-1 / 3}$ remains unchanged since the wave field is measured outside the wave generation area. Including the primary wave flow improves the correlation considerably $\left(R^{2}=0.72\right.$ ), and Eq. 6 proves to be a better estimation of the wave height (Fig. 7).

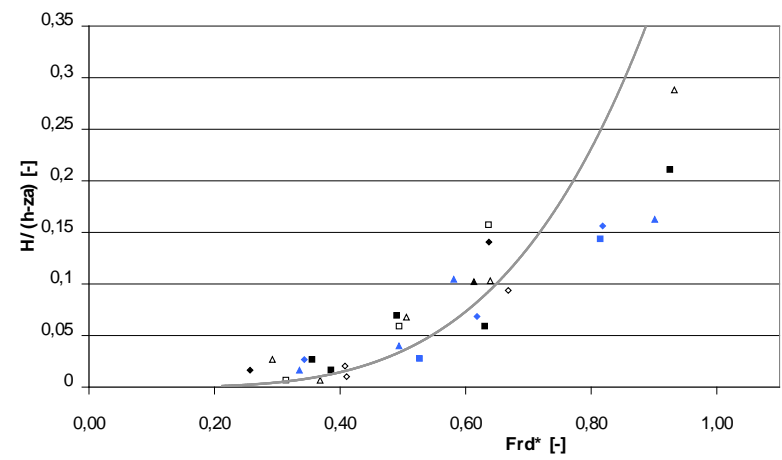

Figure 7. Non dimensional secondary wave height vs hull velocity including primary wave effects (waterlevel depression and return current velocity). Markers represent towing tank data, solid line represents Eq. 6.

Measurements show that the maximum wave height is limited by the water depth, giving an upper boundary to the waveheight $H$ of 0.3 times the waterdepth $h(H \leq 0.3 h)$. At high velocities a stagnant bulge is formed before the hull which streamlines the blunt hull shape. The gradients in the flow hence attain to a maximum and increasing the hull velocity does not result in a larger wave height. Similarly, increasing the blockage $K$ beyond 0.05 did not result in significantly higher secondary waves. 


\section{Conclusions of the physical model study}

The physical model study showed that in contrast to open water cases, the return current highly influences the secondary wave characteristics (wave heights, angles and periods). Since the return flow and water level depression can be well predicted, it is proposed to use the ships velocity relative to the water $V_{h}+U_{r}$ to calculate the wave periods and heights.

\section{NUMERICAL MODEL STUDY}

The physical model results provide a dataset for evaluation of numerical modeling of ship waves in an enclosed environment. The applicability of current state-of-the-art models is evaluated in the following section using a reference towing tank run with moderate hull velocity. Hull blockage $K$ is $13 \%$ and $F_{r, d}$ equals 0.5 . The selected run is on the onset of the trans-critical regime, and a well developed wave pattern can be observed. Secondary wave angles are $60^{\circ}$, measured by using stereo photogrammetry (De Vries 2007). Wave heights of 40-45 mm and wavelengths of $0.6 \mathrm{~m}$ are derived from the timeseries analysis. An overview of the observed wavefield is shown in Fig. 8.

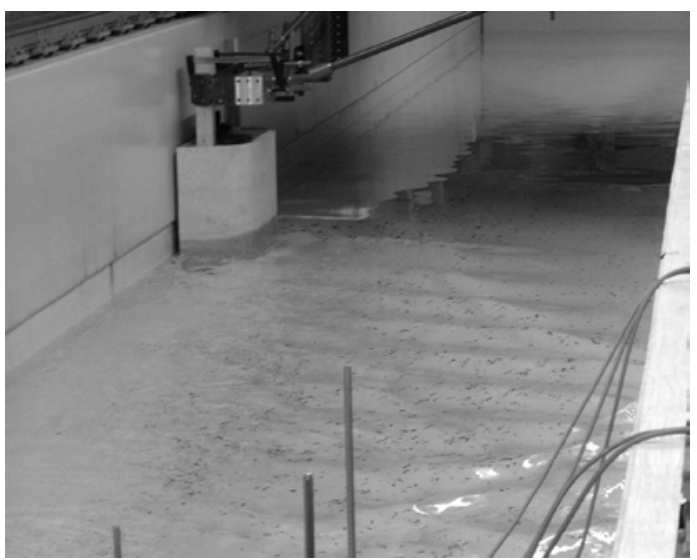

Figure 8: Wave field behind the hull for the reference towing tank run. Note the reduced wave height behind the hull due to the turbulent wake

\section{Panel method modeling}

A common approach to ship wave modeling is using the panel method. Panel method models are based on Green's identity stating that the volumetric differential describing the potential flow can be transformed in a boundary integral of the surfaces. In this paper the DELKELV model (de Koning Gans 1992) is applied.

An overview of the computed wave field behind the hull is given in Fig. 9. Since the panel method is based on potential flow theory the region behind the 
blunt hull affected by flow separation and turbulence is poorly reproduced and the wave height in this region is overestimated.

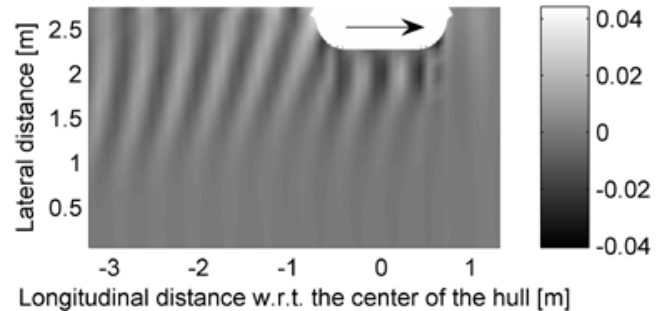

Figure 9: Wave field behind the hull as computed with a panel method model

Wavelengths in the model results are $0.45 \mathrm{~m}$, which is smaller than the measured $0.60 \mathrm{~m}$ but matching the theoretical wavelength for a domain without return current. The primary wave field is underestimated and a water level depression alongside the hull is hardly visible.

Model results are compared to wave gauge records by converting the timeseries into a longitudinal transect using the hulls velocity. In the region alongside the hull the waveheight is underestimated as can be seen from Fig. 10 . The flow in the model is linearized, assuming the magnitude of flow deviations to be small compared to ships velocity. This linearization of the flow, although a sound assumption for slender ships in unrestrained water, suppresses the waterlevel gradients in case of blunt shapes, resulting in an underestimation of wave heights.

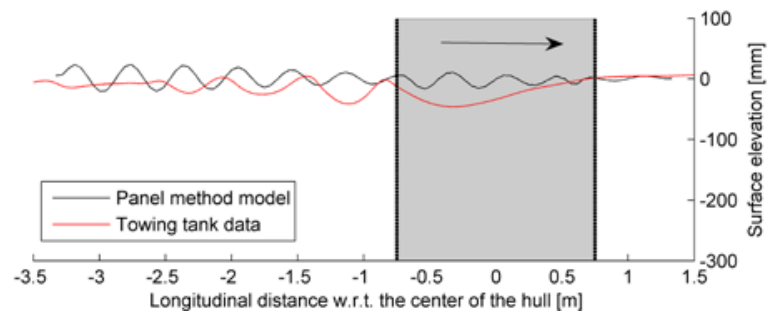

Figure 10: Longitudinal transect at $0.88 \mathrm{~m}$ from the hull.

\section{Finite element method modeling}

The evaluation of panel method modeling underlines the importance of nonlinear effects and vorticity when it comes to correctly reproducing both primary and secondary wave patterns for rather blunt shapes. To incorporate these effects a stabilized finite element model is applied for the incompressible Navier-Stokes equations FINLAB (Labeur and Wells 2007, 2008). The model results for the reference run are presented in Fig. 11 and Fig. 12.

The turbulence in the model causes a decrease in waveheight in the wake of the hull as seen in the towing tank. At the opposite side of the tank, so outside the turbulent wake area, the wave height alongside the hull has the same order 
of magnitude as the measured wave heights, indicating a better representation of the gradients close to the hull. The waterlevel depression and return current alongside the hull are overestimated. It is hypothesized that the mismatch in return current velocity causes the overestimation of the wave angles, wave lengths.

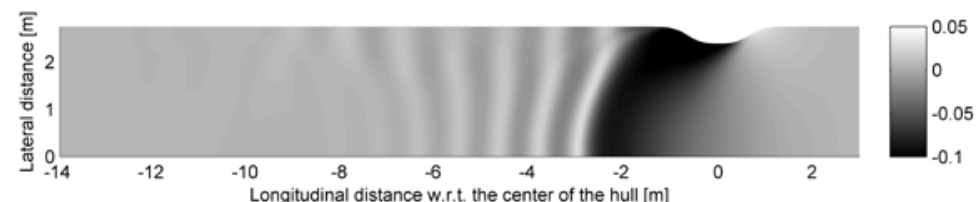

Figure 11: Wave field behind the hull as computed with a FE model

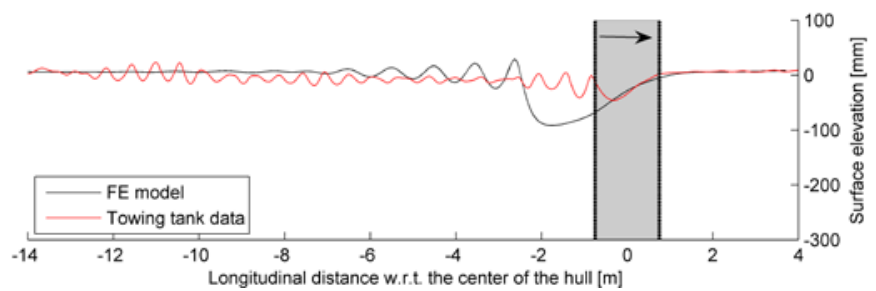

Figure 12: Longitudinal transect at $0.88 \mathrm{~m}$ from the hull.

\section{Conclusions of the numerical modeling}

This first evaluation of these models underlined the difficulties regarding the numerical modeling of the wave pool project, and ship waves in (or close to) the trans-critical regime. Since flow separation and turbulence play such a large role in the wave decay, it is unavoidable to apply complex fluid mechanical models. The small grid and time step size necessary to reproduce the interference pattern makes the complex computations time expensive.

\section{CONCLUSIONS}

We have conducted an experimental study to provide a first assessment of the wave patterns in a circular wave pool. The dataset provides a benchmark for hydrodynamic numerical models in the trans-critical regime. An evaluation of current state of the art models has shown that simulating the combination of a (blunt) fast moving object and wave breaking in a circular enclosure remains challenging, and requires further research.

Overall, we found that the predictive value of theoretical wave height and period assumptions is reasonably good, when applying the ships velocity relative to the water (thus including the return current).

The presented research indicates the importance of currents on the formation of the secondary wave field. Future research on the wave pool concept is to be focused on assessing the currents generated by the continuing movement of the hulls and wave breaking, and the effect on the wave field. 


\section{APPENDIX A: PRELIMINARY WAVE POOL DESIGN}

Based on the results of the towing tank experiment an initial design for the wave pool cross section is presented. The measured maximum wave height over waterdepth ratio $H \leq 0.3 h$ implies that to obtain the required wave height of 2 $\mathrm{m}$ a water depth of at least $7 \mathrm{~m}$ is necessary in the generation area. The transcritical regime should be avoided to prevent translation waves to disturbe the wave field. Furthermore, to attain surfable peel angles on the slope of the inner island the wave angles need to be in the below $75^{\circ}$ in the vicinity of the hull. This leads to an optimum velocity and blockage that can be calculated using Eq. 4 (or Fig. 6), giving $F_{r, d}=0.7$ and $K=0.05$. In prototype scale this corresponds to a hull velocity $V_{h}$ of $5.8 \mathrm{~m} / \mathrm{s}$ and a channel width of $20 \mathrm{~m}$. Wave heights in prototype should attain $2.1 \mathrm{~m}$, based on upscaling of towing tank data with similar blockage and velocity. Using forward wave ray tracing an approximation of the wave crest position and height is calculated including the refraction on the slope (Fig. 13). Calculated peel angles are well within the surfable range.

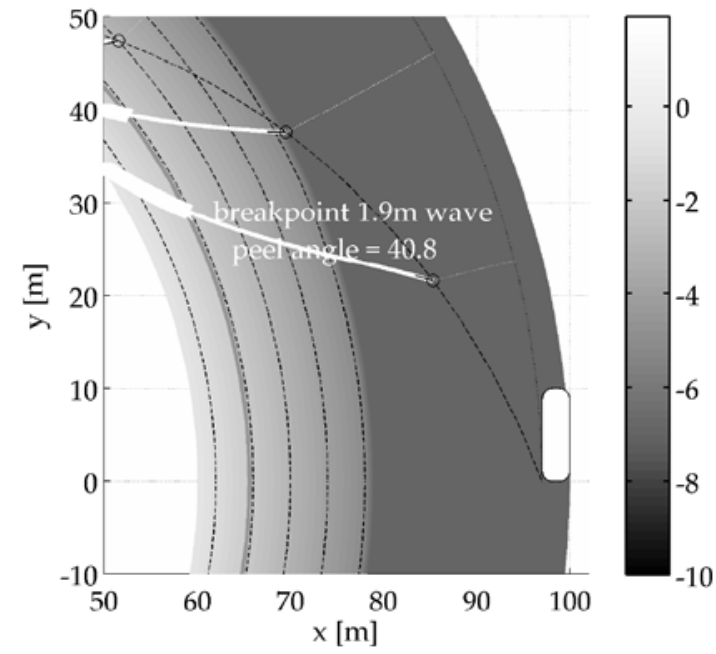

Figure 13: Wave crest refraction in the wave pool. The hull represented by the white rectangle in the lower left corner. Wave crests are indicated by the white lines.

\section{REFERENCES}

Huang, N.E., Z. Shen, S.R. Long, M.C. Wu, H.H. Shih, Q. Zheng, N. Yen, C.C.

Tung and H.H. Liu. 1996. The empirical mode decomposition and the Hilbert Spectrum for nonlinear and non-stationary time series analysis. Proceedings of the Royal Society, London, 903-995. 
Hutt, J.A., K.P. Black and S.T. Mead. 2001. Classification of Surf Breaks in Relation to Surfing Skill. Coastal Research, SI(29), 66-81.

Jansen, P.Ph. and J.B. Schijf. 1953. Influence on the form and dimensions of the cross section of the canal, of the form, of the speed, and the propulsion system of vessels. Proceedings XVIIIth International Navigation Congress, Rome.

Koning Gans, de H. 1992. A higher order panel method for potential flow around an arbitrary body. Delft University of Technology, Delft.

Labeur R.J. and G.N. Wells. 2007. A Galerkin interface stabilization method for the advection-diffusion and incompressible Navier-Stokes equations, Computer methods in applied mechanics and engineering, 196, 4985-5000.

Labeur R.J. and G.N. Wells. In press, accepted 2008. Interface stabilised finite element method for moving domains and free surface flows, Computer methods in applied mechanics and engineering.

Lap, A.J.W. 1954. Fundamentals of ship building and propulsion. International Shipbuilding Progress, Rotterdam.

Schipper, de M.A. 2007. On the generation of surfable ship waves in a circular pool: Part I; Physical background and wave pool design. MSc. Thesis, Delft University of Technology. Delft.

Stoker, J.J. 1957. Water waves; The mathematical theory with applications. Interscience Publishers; New York.

Verheij, H.P. and M.P. Bogaerts. 1986. Sekundaire scheepsgolven en hun invloed op taludbekleding. Verslag modelonderzoek M1115 deel VI (in Dutch), Delft Hydraulics, Delft.

Vries, de S. 2007. On the generation of surfable ship waves in a circular pool: Part II; the application of stereo photo technique measuring water surface elevation and surface flow velocities. MSc. Thesis, Delft University of Technology. Delft.

Walker, J.R. 1974. Recreational surf parameters, Technical report, James K.K. Look Laboratory of Oceanographic engineering, University of Hawaii, 7330 
14

KEYWORDS - ICCE 2008

SURFING WAVES GENERATED BY A HULL

M. A. de Schipper, S. de Vries, M. Henriquez, A.J.H.M. Reniers, H.J. de Koning Gans, R.J. Labeur and M.J.F. Stive

Abstract number 1107

Wave generation

Wave current interaction

Ship waves

Surfing 